\title{
Guest editors' introduction to the ECMLPKDD 2016 journal track special issue of Machine Learning
}

\author{
Thomas Gärtner ${ }^{1}$ • Mirco Nanni ${ }^{2}$. \\ Andrea Passerini $^{3}$. Céline Robardet ${ }^{4}$
}

Published online: 3 August 2016

(C) The Author(s) 2016

This special issue contains papers accepted by the ECMLPKDD 2016 journal track for publication in the Machine Learning journal. Since 2013, the European Conference on Machine Learning and Principles and Practice of Knowledge Discovery has been running the journal track for four consecutive years. To cover the full scope of the conference, journal track papers are published either in this special issue of Machine Learning or in a special issue of Data Mining and Knowledge Discovery. In addition to being published in the respective journal, papers accepted for the journal track are also presented at the conference, just like the papers published in the regular conference proceedings. All accepted papers will be presented by their authors at ECMLPKDD 2016 in Riva del Garda, Italy, during September 19-23, 2016.

To be accepted in the ECMLPKDD journal track, papers needed to be as novel and intriguing as conference papers and at the same time as substantial and mature as journal papers. In total, 58 original manuscripts were submitted to the ECMLPKDD 2016 special issue of Machine Learning, out of which 10 were accepted in time for printing of this special issue. In addition, 2 papers from previous ECMLPKDD journal tracks were included in this special issue, due to a longer reviewing and revision process.

Nayyar Zaidi, Geoffrey Webb, Mark Carman, François Petitjean, and Jesús Cerquides describe an approach to incorporate higher order effects between explanatory variables into Logistic Regression in their paper "ALR ${ }^{n}$ : Accelerated Higher-order Logistic Regression”.

Emanuele Frandi, Ricardo Ñanculef, Stefano Lodi, Claudio Sartori, and Johan A. K. Suykens present and analyse a randomized approach for solving large-scale $\ell_{1}$ regularized regression problems such as Lasso in their paper "Fast and Scalable Lasso via Stochastic Frank-Wolfe Methods with a Convergence Guarantee".

\footnotetext{
Thomas Gärtner

Thomas.Gaertner@nottingham.ac.uk

1 University of Nottingham, Nottingham, UK

2 ISTI - CNR, Pisa, Italy

3 Università degli Studi di Trento, Trento, Italy

4 Université de Lyon, CNRS, INSA de Lyon, LIRIS, Lyon, France
} 
Phong Nguyen, Jun Wang, and Alexandros Kalousis investigate using the ranking algorithm LambdaMART for recommendations by combining it with a low rank factorisation in their paper "Factorizing LambdaMART for cold start recommendations".

Decebal Constantin Mocanu, Elena Mocanu, Phuong H. Nguyen, Madeleine Gibescu and Antonio Liotta present a topological analysis of Restricted Boltzmann Machines and highlight the advantage of constraining networks to scale-free topologies in their paper "A topological insight into restricted Boltzmann machines".

Morteza Haghir Chehreghani proposes a new clustering algorithm based on the trajectory analysis of replicator dynamics for dominant-set graph clustering in his paper "Adaptive Trajectory Analysis of Replicator Dynamics for Data Clustering".

Niall Twomey, Tom Diethe and Peter Flach study the characteristics of sequence classification problems under which directly modelling correlations is superior to indirectly incorporating them in the features of unstructured models in their paper "On the Need for Structure Modelling in Sequence Prediction".

Christian Pölitz, Wouter Duivesteijn and Katharina Morik present a domain adaptation approach based on interpretable low-dimensional projections in their paper "Interpretable Domain Adaptation via Optimization over the Stiefel Manifold".

Christian Daniel, Herke van Hoof, Jan Peters and Gerhard Neumann propose a probabilistic formulation of the option framework for Reinforcement Learning applicable to both continuous and discrete settings in their paper "Probabilistic Inference for Determining Options in Reinforcement Learning".

Nikolaos Nikolaou, Narayanan Unny Edakunni, Meelis Kull, Peter Flach and Gavin Brown report an extensive theoretical and experimental study of the existing literature on costsensitive boosting algorithms in their paper "Cost-sensitive boosting algorithms: Do we really need them?".

Uwe Dick and Tobias Scheffer present a structured-output learning algorithm for HTTPLayer DDoS attacker detection which joinly optimises the structured-prediction model and the controller used to search for the correct output in their paper "Learning to Control a Structured-Prediction Decoder for Detection of HTTP-Layer DDoS Attackers".

Shalmali Joshi, Joydeep Ghosh, Mark Reid, and Oluwasanmi Koyejo propose and study an approach for clustering in multiple views based on using Rényi divergence as a coregularization term in their paper "Rényi Divergence Minimization based Co-regularized Multiview Clustering".

Ranganath B. N. Swamy proposes an alternating minimisation based approach to explore sparse relationships in topic models in the paper "Sparse Topical Analysis of Dyadic Data using Matrix Tri-factorization".

This special issue would not have been possible without the help of many people: we would like to say many thanks to all authors of submitted papers, the active members of the ECMLPKDD 2016 guest editorial board, and all additional reviewers. Many thanks go also to the other chairs of ECMLPKDD 2016, it was a pleasure organising the conference with you! Further thanks go to the KU Leuven for hosting RAFT, to Thanh Le Van for developing RAFT, and to Anandhi, Janaki, Juffi, Melissa, Peter, and Venkat for their tireless help and support of the journal track. 